\title{
PROSPECTIVE STUDY OF MANAGEMENT OF FRACTURE NECK OF FEMUR BY HEMIARTHROPLASTY WITH CEMENTED BIPOLAR
}

\author{
Hanu Tej Adapureddi ${ }^{1}$, S. B. Kamareddy², Anand Kumar ${ }^{3}$, Sri Krishna Paturi ${ }^{4}$, Sandeep Anne ${ }^{5}$, Jaya Prakash Reddy \\ 1 Post Graduate, Department of Orthopaedics, Basaveswar Teaching and General Hospital. \\ 2 Professor, Department of Orthopaedics, Basaveswar Teaching and General Hospital. \\ ${ }^{3}$ Post Graduate Department of Orthopaedics, Basaveswar Teaching and General Hospital. \\ ${ }^{4}$ Post Graduate, Department of Orthopaedics, Basaveswar Teaching and General Hospital. \\ 5 Post Graduate, Department of Orthopaedics, Basaveswar Teaching and General Hospital. \\ ${ }^{6}$ Senior Resident, Department of Orthopaedics, Basaveswar Teaching and General Hospital.
}

\section{ABSTRACT}

\section{BACKGROUND AND OBJECTIVES}

Fracture neck of femur is a leading cause of hospital admissions in elderly age group. The number of such admissions is on a rise because of increased longevity, osteoporosis and sedentary habits. Conservative methods of treatment is not acceptable because it results in nonunion with unstable hip and limitation of hip movement as well as complications of prolonged immobilization like bed sores, deep vein thrombosis and respiratory infections. Hemiarthroplasty remains the most common modality of treatment in our country. The time-tested unipolar prosthesis is being slowly replaced by bipolar prosthesis, which is claimed to have a lower incidence of complications. This study was conducted to analyze the results of surgical management of fracture neck of femur using modular bipolar hemiarthroplasty.

\section{MATERIALS AND METHODS}

The present study was a prospective study of 50 cases of fracture neck of femur admitted to Basaweshwar Hospital attached to MR Medical College, Kalburagi, between the study periods of July 2014 to July 2015. Cases were selected according to inclusion and exclusion criteria, i.e., patients with intra-capsular fracture neck of femur above the age of 55yrs. Medically unfit and patients not willing for surgery were excluded from the study.

\section{RESULTS}

In our series of 50 cases, there were 19 males and 31 females with a maximum age of 92 yrs, minimum age of 58 yrs, and an average age of 65 years. There was a slight predominance of left-sided fractures when compared to the right. The mean duration of hospital stay was 5 days. At the final one year follow-up assessment with Harris Hip Score, 18 patients (35\%) achieved 'Excellent' result, 22 patients (45\%) achieved 'Good' result, 5 patients (10\%) achieved 'Fair' result and 5 patients (10\%) achieved 'Poor' result. Overall, $80 \%$ of the patients achieved an excellent or good result. On enquiry regarding the overall satisfaction with the procedure and return to pre-fracture levels of activity, 18 patients (35\%) were 'Very Satisfied,' 25 (50\%) were 'Fairly Satisfied' and 7 (15\%) were 'Not Satisfied.'

\section{CONCLUSION}

Modular bipolar hemiarthroplasty for fractures of the femoral neck provides better range of movement, freedom from pain and more rapid return to unassisted activity with an acceptable complication rate. The end functional results depend on the age of the patient, associated co-morbidity and optimum post-operative rehabilitation. The advantage of the system is in the modularity obtained from the different sized stems, shell which are available in increments of size allow exact matching of the head and the ease with which the system can be converted to total hip arthroplasty without replacing the femoral stems. The long-term results using modular bipolar hemiarthroplasty needs further study for a longer period in a larger sample.

\section{KEYWORDS}

Fracture neck femur; Elderly; Hemiarthroplasty; Modular Bipolar Prosthesis; Harris Hip Score.

HOW TO CITE THIS ARTICLE: Hanu Tej Adapureddi, S. B. Kamareddy, Anand Kumar, Sri Krishna Paturi, Sandeep Anne, Jaya Prakash Reddy. "Prospective Study of Management of Fracture Neck of Femur by Hemiarthroplasty with Cemented Bipolar." Journal of Evolution of Medical and Dental Sciences 2015; Vol. 4, Issue 98, December 07; Page: 16309-16314, DOI: $10.14260 /$ jemds/2015/2407

Financial or Other, Competing Interest: None.

Submission 31-10-2015, Peer Review 02-11-2015,

Acceptance 13-11-2015, Published 04-12-2015.

Corresponding Author:

Dr. Hanu Tej Adapureddi,

Post Graduate, Department of Orthopaeadics,

Basaveswar Teaching and General Hospital.

Kalburgi.

E-mail: hanutej.surya@gmail.com

DOI:10.14260/jemds/2015/2407

\section{INTRODUCTION}

Femoral neck fractures, one of the most common injuries in the elderly have always presented great challenges to orthopaedic surgeons. The prevalence of these fractures has increased with improvement in life expectancy, increased incidence of osteoporosis, poor vision, neuro-muscular incoordination and changes in lifestyle leading to sedentary habits. 
The incidence of these fractures are expected to double in the next twenty years and triple by the year 2050. ${ }^{1}$ The prevalence of the fracture also doubles for each decade of life after the fifth decade. ${ }^{2}$

With our society becoming more and more a geriatric society, the burden of this fracture and its sequelae continues to be on the rise. ${ }^{3}$ The goal of treatment of femoral neck fractures is restoration of pre-fracture function without associated morbidity. ${ }^{4}$ However, treatment of displaced femoral neck fractures in elderly has been controversial. Open reduction and internal fixation of these fractures in elderly has poor outcome including high rate of non-union and avascular necrosis.

Bateman in 1974 introduced the Bipolar prosthesis which had mobile head element and had additional head surface to allow movement within the acetabulum. This led to reduced wear of acetabular surface and hence reduced incidence of pain and acetabular protrusion because motion is present between the metal head and the polyethylene socket (Inner bearing) as well as between the metallic head and acetabulum (Outer bearing). 5

Initially, the Bipolar prostheses were of non-modular design followed presently by the modular prostheses. The modular nature of the prosthesis allows for neck length adjustment with interchangeable stems. Future conversion to a total hip replacement is easier with a modular prosthesis, because only the acetabular component needs to be added. The advantage of the system is in the modularity obtained from the different sized stems, shell which are available in increments of size allow exact matching of the head and the ease with which the system can be converted to total hip arthroplasty without replacing the stems. ${ }^{6}$

Bipolar prosthesis is slowly replacing the conventional unipolar prosthesis in the ever increasing segment of 'Active elderly' because of its superior benefits and its attractive pricing. ${ }^{7,8}$ Its advantages over unipolar endoprosthesis are higher percentage of satisfactory results, less post-operative pain, greater range of movements, more rapid return to unassisted activity and reduced incidence of acetabular erosion. $7,8,9$

We have taken up this study to gain a deeper understanding of the results and problems associated with this procedure.

\section{OBJECTIVES}

1. To study the functional outcome of intracapsular fracture of femoral neck with modular bipolar prosthesis in Indian population.

2. To study the end results of modular bipolar prosthesis with respect to pain, mobility and stability.

3. To study the complications of modular bipolar hemiarthroplasty.

\section{SOURCE OF DATA}

Patients who have sustained an intracapsular femoral neck fracture and are admitted to Basaweshwar Hospital attached to MR Medical College, Kalaburagi will be taken for this study after obtaining their consent. This is a prospective study from July 2014 to July 2015.

No. of cases: 50 cases.

\section{Inclusion Criteria}

- Patients of age between 55-65yrs with displaced intracapsular fracture neck of femur, in whom osteosysthesis was expected to give unsatisfactory results because of various reasons like late presentation and nonunion.

- Failed internal fixation of fracture neck of femur

- Patients of >65 yrs. age having radiologically normal appearing acetabulum and not affordable for primary total hip replacement at the time of presentation.

\section{Exclusion Criteria}

- Patients below 55 years.

- Patients with arthritic changes involving the acetabulum.

- Pathological fractures.

- Patients not willing for surgery.

- Patients medically unfit for surgery.

\section{Method of Collection of Data}

Patients with fracture neck of femur satisfying the inclusion criteria, who required surgical intervention, were worked up clinically and radiologically. All patients selected for the study were examined according to protocol, associated injuries, if any, were noted and investigations carried out in order to evaluate fitness for anesthesia.

\section{Preoperative Protocol}

All study patients were put on skin traction and 4-7 kilograms of weight applied to maintain the length of the lower limb and facilitate subsequent hemiarthroplasty procedure. Adequate medical management of associated comorbid conditions like Diabetes Mellitus, Systemic Hypertension, Chronic Obstructive Pulmonary Disease and Heart Diseases were initialized to optimize patient's fitness for anesthesia. An informed written consent for the procedure as per the guidelines of the institution and a consent for inclusion of the patient for the present study was taken. The peri-operative antibiotic used was cefoperazone sulbactam $1.5 \mathrm{~g} 12^{\text {th }}$ hourly intra-venous starting 20 minutes before the procedure and continued for 5-7 days. None of the study patients received Deep Vein Thrombosis (DVT) prophylaxis.

\section{Follow Up}

Regular follow up of all cases was done at 6 weeks, 3 months, 6 months, 9 months and one year. At each followup, patients were evaluated clinically using the Harris Hip Score 100 and radiologically with appropriate x-rays.

\section{THE HARRIS HIP SCORE}

Maximum points possible - 100

- $\quad$ Pain relief, 44 points

- $\quad$ Function, 47 points

Gait (33 possible points) Activities (14 possible points)

- $\quad$ Range of motion, 5 points

- Absence of deformity, 4 points 


\section{Pain (44 possible)}

a) None or ignores it, 44 points

b) Slight, occasional, no compromise in activities, 40 points

c) Mild pains, no effect on average activities, rarely moderate pain with unusual activity, may take aspirin 30 points.

d) Moderate pain, tolerable, but makes concessions to pain with some limitation of ordinary activity or work, 20 points.

e) Marked pain, serious limitation of activities, 10 points.

f) Totally disabled, crippled, pain in bed, bed ridden, 0 points.

\section{Function (47 possible)}

A) Gait (33 possible)

i) Limp
a) None 11 points
b) Slight 8 points
c) Moderate 5 points
d) Severe 0 points

ii) Support
a) None 11 points
b) Cane for long walk 7 points
c) Cane most of the time 5 points
d) One crutch 3 points
e) Two canes 2 points
f) Two crutches 0 point
g) Not able to walk (Specify reason) 0 point

iii) Distance walked
a) Unlimited 11 points
b) About 1000 meters, 8 points
c) About 5000 meters, 5 points
d) Indoors only 2 points
e) Bed and chair 0 point

\section{B) Activities (14 possible points)}

i) Stairs (4 maximum)

a) Foot over foot without use of banister, 4 points

b) Foot over foot using banister, 2 points

c) Stairs in any manner, 1 point

d) Unable to do stairs, 0 point

ii) Shoes and socks (4 maximum)

a) With ease 4 points

b) With difficulty 2 points

c) Unable, 0 points

iii) Sitting

a) Comfortably in ordinary chair for one hour, 5 points

b) On a high chair for half an hour, 3 points

c) Unable to sit comfortably in any chair, 1 point

iv) Ability to enter public transportation, 1 point

Absence of Deformity: Points (4) are given if the patient demonstrates:

a) Less than 300 fixed flexion contracture

b) Less than 100 fixed adduction

c) Less than $10^{\circ}$ fixed internal rotation in extension

d) Limb length discrepancy less than $3.2 \mathrm{~cm}$
Range of Motion (5 points possible)

\begin{tabular}{|c|c|c|c|c|}
\hline $\begin{array}{c}\text { Hip } \\
\text { Movement }\end{array}$ & Range & Multiplying & $\begin{array}{c}\text { Patient's } \\
\text { Range }\end{array}$ & Score \\
\hline Flexion & $0-45$ & 1 & & \\
\hline & $45-90$ & 0.6 & & \\
\hline & $90-110$ & 0.3 & & \\
\hline Abduction & $0-15$ & 0.8 & & \\
\hline & $15-20$ & 0.3 & & \\
\hline Adduction & $0-15$ & 0.2 & & \\
\hline $\begin{array}{c}\text { ER in } \\
\text { extension }\end{array}$ & $0-15$ & 0.4 & & \\
\hline $\begin{array}{c}\text { IR in } \\
\text { extension }\end{array}$ & any & 0 & & \\
\hline TOTAL & & & & \\
\hline
\end{tabular}

\section{RESULTS}

During the period between July 2014 to July 2015, 50 patients were treated with modular bipolar hemiarthroplasty for fracture neck of femur at the Basaweshwar Hospital attached to MR Medical College, Kalburagi. Data was collected based on detailed patient evaluation with respect to history, clinical examination and radiological examination. The postoperative evaluation was done both clinically and radiologically. Out of the 50 cases all patients were available for followup till one year, which was taken as a basic pre-requisite for inclusion in the study. In our series of 50 cases, there were 19 males and 31 females. Youngest was of age 55 yrs and oldest 85 yrs. Average age was 65 yrs. Left sided fractures were 33 (65\%), more common than right.

Trivial fall history was given by 35 cases $(70 \%)$. Radiologically transcervical fractures were 40 cases (80\%), basicervical 8 cases and subcapital 2 cases. Peri-operatively technical difficulty was faced in 5 cases, intraop hypotension after putting cement occurred in 3 and postop hypotension in 2 patients. Early postop complications like limb shortening were seen in 5 cases and surgical site infection in 3 cases. The minimum duration of hospital stay amongst the study patients was 7 days and maximum duration was 20 days with the average being 10 days.

All patients were followed up regularly at 6 weeks, 3 months, 6 months, 9 months and one year. Only the patients who completed a one-year follow-up were included in the final analysis. The Harris Hip Scores were recorded at each follow-up visit.

FINAL HARRIS HIP SCORE AND CLINICAL RESULT

\begin{tabular}{|c|c|c|c|}
\hline Grade & $\begin{array}{c}\text { Harris hip } \\
\text { Score }\end{array}$ & $\begin{array}{c}\text { No. } \\
\text { of Pts. }\end{array}$ & $\begin{array}{c}\text { Percentage } \\
\text { (\%) }\end{array}$ \\
\hline Excellent & $90-100$ & 18 & 35 \\
\hline Good & $80-89$ & 22 & 45 \\
\hline Fair & $70-79$ & 5 & 10 \\
\hline Poor & $<70$ & 5 & 10 \\
\hline
\end{tabular}


COMPARISON OF OUR CLINICAL

\section{RESULT WITH STANDARD STUDIES}

\begin{tabular}{|c|c|c|c|}
\hline Grade & Our Study & $\begin{array}{c}\text { Moshein } \\
\mathbf{1 0 3}\end{array}$ & $\begin{array}{c}\text { Last Range } \\
\text { Study 104 }\end{array}$ \\
\hline Excellent & 35 & 40 & 39.6 \\
\hline Good & 45 & 25 & 31.2 \\
\hline Fair & 10 & 23 & 15.3 \\
\hline Poor & 10 & 12 & 13.9 \\
\hline
\end{tabular}

Case-1

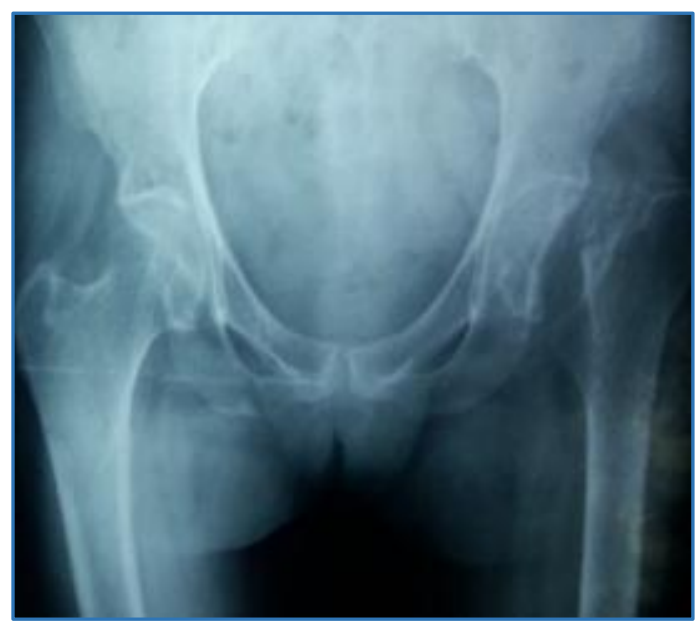

Pre-Operative

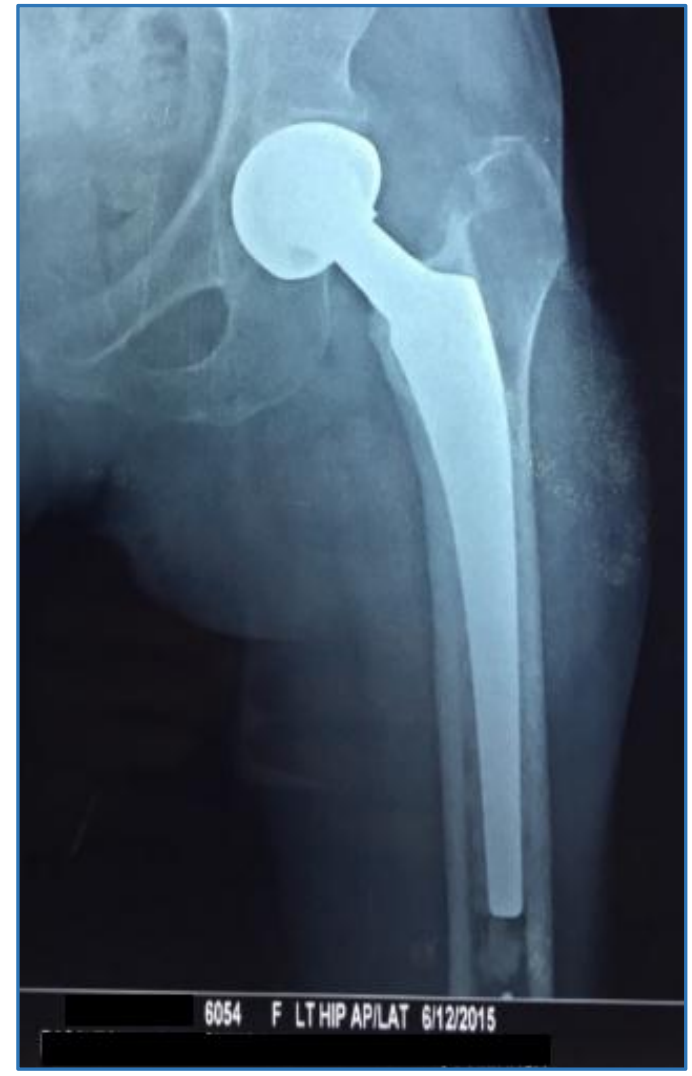

Post-Operative
Case 2

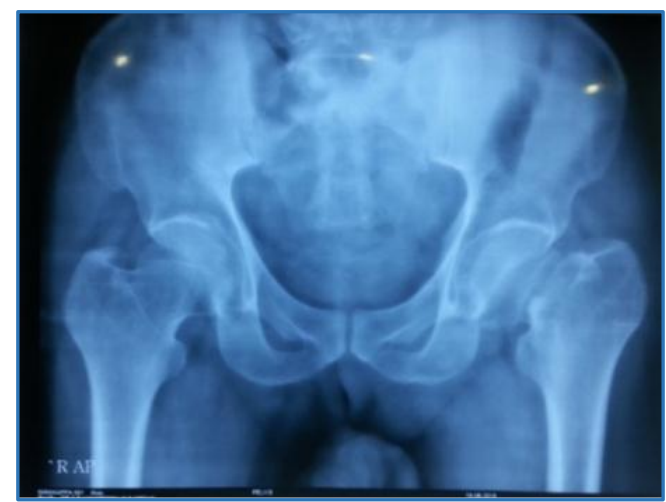

Pre-Operative

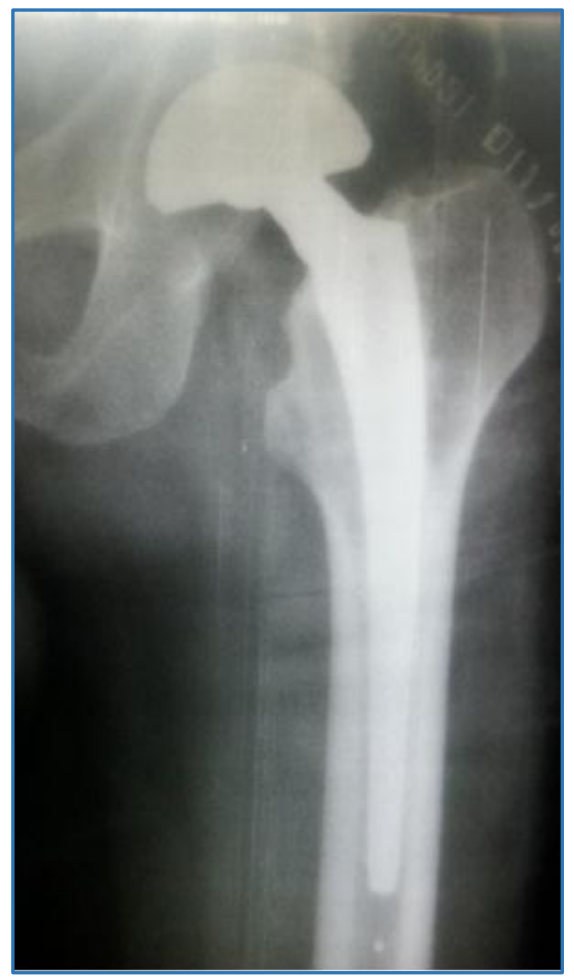

Post-Operative

\section{DISCUSSION}

The aim of replacement surgery in trans-cervical fracture neck femur is early return to daily activities. This is particularly applicable to the elderly age group where complications need to be prevented. The mean age of the patients in the present study was 65 years. The aim of assessing age is to estimate the patient's mean survival time and their ability to comply with rehabilitation protocol. Patients with hip fractures have an increased mortality rate during the first year after fracture, but after one year the mortality rate is comparable to that of the general population. The results of our study showed that age of the patient had minimal influence on the final clinical result.

As in most standard studies, the present study also had a higher number of females with the left side more commonly affected than the right. Majority of our study patients $(70 \%)$ sustained the injury due to a trivial trauma like tripping or slipping. This is a very common occurrence in elderly population where poor vision and lack of neuromuscular coordination is a problem. 
Most of such injuries can be classified as "Indirect" trauma. Other patients (30\%) due to a Road Traffic Accident.

A little more than half of our study patients were brought to the hospital within three days of sustaining the injury; $25 \%$ of the patients were brought to the hospital within 24 hours of the injury while $30 \%$ presented for treatment within $24 \mathrm{hrs}$. to $72 \mathrm{hrs}$. One-sixth of our study patients (15\%) were brought to the hospital between $72 \mathrm{hrs}$ to $1 \mathrm{wk}$. and the remaining $15 \%$ presented for treatment after one week and $15 \%$ of patients after 3 wks. This is a common scenario in our country where patients present to a doctor much late given the seriousness of the condition. Difficulty in post-operative rehabilitation was particularly noticed in the subset who presented after 1 week.

All of our study patients had a displaced fracture of the neck of femur. Majority of the patients (80\%) had a transcervical fracture while 8 patients had a basicervical and 2 had sub-capital fracture. The anatomical type of fracture and the displacement did not have any bearing on the final function. Diabetes was found to be the most common comorbidity seen in $25 \%$ of the study patients. All patients had Type II Diabetes and were on oral hypoglycaemic agents. They were shifted to insulin pre-operatively and blood sugar values optimized before taking up for surgery. The other comorbid conditions seen in the order of frequency were hypertension (25\%), COPD (20\%), heart disease and ipsilateral knee osteoarthritis (10\%). It was observed that the postoperative rehabilitation of patients was significantly affected by the presence of the above co-morbidities. ${ }^{10}$ This also had an effect on the final functional result of the procedure. ${ }^{11}$ Similar observations have been made by Koval et al. and Bath. $12,13,14$

Delay in taking up for surgery was usually for optimizing the medical condition of the patient. All cases were performed on an elective basis and were scheduled as the first surgery in the morning.

All the surgeries were performed under spinal or epidural anaesthesia after a thorough preanaesthetic evaluation and preparation. The choice of the type of anesthesia was as per the anesthetist's discretion.

All patients were operated after being put into lateral decubitus position by the posterior approach of Moore. The posterior approach was preferred because of the familiarity of most of the surgeons at our institution with the approach. Though the dislocation rate is reported to be more with the posterior approach, none of our study patients had a postoperative dislocation of the prosthesis.8,15 This was because meticulous attention was given to suturing the posterior capsule and the short external rotators and keeping the limb in slight abduction after the procedure. Patients were also explained in the immediate postoperative period about the risk of dislocation with excessive flexion or adduction of the hip.

The size of the prostheses used in general matched well with the preoperatively measured size of the head as assessed by x-rays. In $40 \%$ of the cases $45 \mathrm{~mm}$ prostheses were used. This was followed in frequency by $43 \mathrm{~mm}(20 \%)$, $47 \mathrm{~mm}(20 \%)$ and $41 \mathrm{~mm}(10 \%)$ prostheses in the order of frequency. The rasps used for broaching the canal were part of the instrumentation that came with the prosthesis.

The advantage was that the rasp corresponded to the exact length and width of the prosthesis which prevented any additional rasping of the canal and subsequent loose seating of the prosthesis. All the prostheses were inserted by press fit technique.

Additional augmentation of femoral stem with cement was used in patients with osteoporosis, wide medullary canal and without serious medical comorbidities. Technical difficulties encountered with the procedure were most often related to the operating surgeons' learning curve. The main difficulty faced was calculating the angle of the neck osteotomy, which in the case of the bipolar prosthesis was more vertical as compared to the traditional Austin Moore's prosthesis. This resulted in poor seating of the prosthesis collar on the neck and calcar. The second difficulty encountered was difficulty in reduction with proper size prosthesis in old, neglected fracture neck of femur cases because of soft tissue contracture even though after putting on skeletal traction. In upto $60 \%$ of the cases, the blood loss was $<500 \mathrm{ml}$ for the whole procedure and in most of the others it was between 500 to $750 \mathrm{ml}$. Only $15 \%$ of cases had a blood loss of $>750 \mathrm{ml}$ requiring a blood transfusion. It has been reported in literature that the average blood loss with hip hemiarthroplasty is less in the anterior approach as compared to the posterior approach. ${ }^{15,17}$ Most of the surgeries were completed between 90-120 minutes of starting the procedure. Similar duration of the procedure has been reported by Haidukewych, et al. ${ }^{9}$ and Drinker, et al. ${ }^{16} 17$ Neither the intraoperative blood loss nor the duration of the procedure had any effect on final function. Most of our study patients were mobilized in bed on day one of surgery and with weightbearing as tolerated within the 72 hours postoperative period. Delay if at all was due to medical reasons. Persisting of limb shortening $(1-1.5 \mathrm{~cm})$ was observed in two patients of old neglected fractures who had gross shortening $(6-7 \mathrm{~cm})$ preoperatively and effectively managed with heel risers. Superficial infection in the form of a wound dehiscence was seen in 3 patients (5\%); 2 patients were managed by antibiotics alone after culture sensitivity and 1 patient by debridement and secondary suturing with adequate control of the diabetic status and appropriate antibiotics based on culture-sensitivity results.

The infection resolved without any sequelae and there was no late reactivation of the same. Infection rate of 3.9\% after bipolar hemiarthroplasty is reported by Nottage, et al. ${ }^{18}$ None of our study patients had bed sores. The minimum duration of hospital stay amongst the study patients was 7 days and maximum duration was 20 days with the average being 7 days. Average hospital stay of 21 days with bipolar hemiarthroplasty has been reported by Lestrange. Drinker and Murray have reported an average hospital stay of 23 days with the same procedure. ${ }^{16}$ There were no late postoperative complications like loosening, dislocation, erosion, secondary osteoarthritis, protrusio acetabuli or periprosthetic fracture. We are unable to comment upon long-term acetabular erosion due to relative short followup. All patients were followed up regularly at 6 weeks, 3 months, 6 months, 9 months and one year. Only the patients who completed a one- year follow-up were included in the final analysis. The Harris Hip Scores were recorded at each follow-up visit. In our study, the final Harris Hip Score as evaluated at one year follow-up averaged 85.68 with the maximum score being 93 and the minimum score being 65.8 . Overall, 18 patients (35\%) achieved excellent result, 22 patients (45\%) achieved good result, 5 patients (10\%) achieved fair result and 5 patients $(10 \%)$ achieved poor 
result. Overall, $80 \%$ of the patients achieved an excellent or good result.

Our results are comparable with standard studies of bipolar hemiarthroplasty performed for fracture neck femur. Poor results was observed in patient with old fracture neck of femur.

All study patients were also evaluated with their level of satisfaction with the procedure and their ability to return to pre-fracture level of activity; 18 patients (35\%) were 'very satisfied,' 25 (50\%) were 'fairly satisfied' and 7 (15\%) were 'not satisfied.' The level of satisfaction being a subjective assessment did not correlate well with the Harris Hip Score, which was an objective assessment. Our study is not without its own shortcomings. Firstly, our duration of follow-up of one year is very less in assessing the longevity and functional endurance of the prosthesis used and hence come to definitive conclusions. Secondly, we have not evaluated the degree of intraprosthetic motion at the inner bearing at each follow-up. Such studies are complicated and beyond the facilities available at our institution. Such studies are indicated because there are claims that the motion at the inner bearing reduces over time and most prostheses behave as unipolar prostheses over a period of time.

\section{CONCLUSION}

Modular Bipolar hemiarthroplasty for fractures of the femoral neck provides better range of movement, freedom from pain and more rapid return to unassisted activity with an acceptable complication rate. The end functional results depend on the age of the patient, associated comorbidity and optimum postoperative rehabilitation. Though out of the purview of the present study, our experience with modular bipolar prosthesis have been significantly better than that with Austin Moore's prosthesis. The advantage of the system is in the modularity obtained from the different sized stems and neck which is available in different sizes; and the ease with which the system can be converted into total hip arthroplasty without replacing the femoral stem. Since this study duration is short, none of the patient was subjected to total hip replacement. The long term results using modular bipolar hemiarthroplasty needs further study for a longer period in a larger sample.

\section{BIBLIOGRAPHY}

1. Schmidt AH, Swiontkowski MF. Femoral neck fractures. Orthop Clin North Am 2002; 33(1): 97-111.

2. Leighton RK: Fractures of the Neck of the Femur. In: Rockwood and Green's fracture in Adults. Ed: Bucholz RW, Heckman JD, Court-Brown CM. 6th edn. Philadelphia, Lippincott Williams \& Wilkins 2006; 1753-1791.

3. Swiontowski MF. Intracapsular fractures of the hip. J Bone Joint Surg Am 1994; 76: 129-138.

4. Ioro R, Healy WL, Lemos DW, Appleby D, Lucchesi C, Saleh KJ, et al. Displaced femoral fractures in the elderly: outcomes and cost effectiveness.Clin Orthop 2001; 383: 229-242.
5. Bhandari M, Devereaux PJ, Swiontowski MF, Tornetta P, Obremskey W, Koval KJ, et al. Internal fixation compared with arthroplasty for displaced fractures of the femoral neck. J Bone Joint Surg Am 2003; 85:1673-1681.

6. Ioro R, Schwartz B, Macaulay W, Teeney SM, Healey WL, York S. Surgical treatment of displaced femoral neck fractures in the elderly: a survey of the American Association of Hip and Knee Surgeons. J Arthroplasty 2006;21(8): 1124-1133.

7. Agarwala S, Bhagwat A. Charnley-Hasting bipolar modular replacement option for Intracapsular fracture neck of femur. Indian J Ortho 2006; 40(3):157-159.

8. Marya SK, Thukral R, Hasan R, Tripathi M. Cementless bipolar hemiarthroplasty in femoral neck fractures in elderly. Indian J Orthop 2011; 45:236-242.

9. Bednar JM, Friedenberg ZB, Turner ML. Bipolar femoral endoprosthesis: A study correlating component movement with clinical outcome. J Trauma 1988;28:664670.

10. Bateman JE, Berenji AR, Bayne 0, Greyson ND. Long term results of bipolar hemiarthroplasty in osteoarthritis of the hip. Clin Orthop 1990;251:54 -66.

11. Vazquez-Vela G, Vazquez-Vela E, Dobarganes FG. The Bateman bipolar prosthesis in osteoarthritis and rheumatoid arthritis - A review of 400 cases. Clin Orthop 1990;251:82 -91.

12. Harris WH. Traumatic arthritis of the hip after dislocation and acetabular fractures: Treatment by mould arthroplasty. An end result study using a new method of result evaluation. J Bone Joint Surg Am 1969;51-A(4): 737-755.

13. LaBelle LW, Colwill JC, Swanson AB. Bateman bipolar hip arthroplasty for femoral neck fractures - A five to ten year follow up study. Clin Orthop 1990;251:20-25.

14. Moshein J, Alter AH, Elconin KB, Adams WW. Transcervical fractures of the hip treated with the bateman bipolar prosthesis. Clin Orthop 1990;251:4853.

15. Lestrange NR. Bipolar arthroplasty for 496 hip fractures. Clin Orthop 1990;251:7 -18.

16. Koval KJ, Zuckerman JD. Current Concepts Review: Functional Recovery after Fracture of the Hip. J Bone Joint Surg Am 1994;76:751-766.

17. Bath, R. Problems in the treatment of femoral neck fractures. Proceedings of the Royal Society of Medicine 1975; 63:1120-1128.

18. Drinker H, Murray WR. The universal proximal femoral endoprosthesis - A short term comparison with conventional hemiarthroplasty. J Bone Joint Surg 1979;61A:1167-1174. 